\title{
Main lesions in the central nervous system of dogs due to Leishmania infantum infection
}

\author{
Weline Lopes Macau', Joicy Cortez de Sá2, Ana Patrícia de Carvalho da Silva ${ }^{3}$, Alessandra Lima Rocha ${ }^{4}$, \\ Renata Mondêgo-Oliveira ${ }^{4}$, Fábio Henrique Evangelista de Andrade ${ }^{4}$, Caroline Magalhães Cunha ${ }^{5}$, \\ Kátia da Silva Calabrese ${ }^{5}$ and Ana Lucia Abreu-Silva ${ }^{4^{*}}$ (D)
}

\begin{abstract}
Background: Canine visceral leishmaniasis (CVL) is endemic in São Luís Maranhão/Brazil and it leads a varied clinical picture, including neurological signs.

Results: Histopathological evaluation showed that 14 dogs exhibited pathological alterations in at least one of the analyzed areas. Of these, mononuclear inflammatory reaction was the most frequent, although other lesions, such as hemorrhage, chromatolysis and gliosis were also observed. The presence of $L$. infantum amastigotes was confirmed in eight dogs, identified in four regions: telencephalon, hippocampus, thalamus and caudal colliculus, but only one presented neurological signs. Polymerase chain reaction results detected the DNA of the parasite in 11 samples from seven dogs. The positive areas were the telencephalon, thalamus, hippocampus, cerebellum, caudal and rostral colliculus.
\end{abstract}

Conclusion: These results reveal that during canine visceral leishmaniasis, the central nervous system may display some alterations, without necessarily exhibiting clinical neurological manifestations. In addition, the L. infantum parasite has the ability to cross the blood brain barrier and penetrate the central nervous system.

Keywords: Dog, Encephalon, Histopathology, Immunohistochemistry, Leishmania infantum, Polymerase chain reaction

\section{Background}

Canine visceral leishmaniasis (CVL) is a chronic and progressive disease, characterized by the dissemination of the parasite through various organs. The amastigote forms of Leishmania, however, are found mostly in the mononuclear phagocytic system organs, such as the spleen, lymph nodes, liver and bone marrow [1-4].

The blood-brain barrier (BBB) is a dynamic and functional structure that separates the central nervous system from the systemic circulation and is required for maintaining neuronal function [5]. It has been believed for some time that Leishmania parasites were unable to cross the BBB. However, in 1996, the presence of Leishmania

\footnotetext{
* Correspondence: anabreu@uema.br

${ }^{4}$ Department of Pathology, Universidade Estadual do Maranhão, São Luís, MA CEP 65055-000, Brazil

Full list of author information is available at the end of the article
}

donovani amastigote forms in the cerebrospinal fluid (CSF) was described for first the time in a human patient [6]. Since then, the central nervous system has become the subject of many studies seeking to confirm that Leishmania species cross the BBB and cause pathological alterations and clinical neurological signs [8, 10, 11, 20, 22].

A study with dogs positive for visceral leishmaniasis, demonstrated the presence of anti-Leishmania antibodies in the CSF, that alterations in the nervous tissue of these animals can occur and this may result in a form of the disease known as "cerebral leishmaniasis" [7]. Experimental studies in mice infected with Leishmania amazonensis, associated with the cutaneous form of leishmaniasis in humans, described the occurrence of brain lesions with discrete hyperemia and inflammatory infiltrates in the meninges, composed of mononuclear cells and neutrophils [8]. 
Another study using an experimental model of infection in hamsters with Leishmania sp. identified the dissemination of the parasite in a number of organs. The main finding of the study was the high percentage of animals with parasites in the central nervous system [9].

Neurological signs following natural infection in dogs were described in a case of acute paraplegia associated with vasculitis [10]. In this study, post-mortem examination revealed a large area of hemorrhaging on the nervous tissue of the spinal cord, together with thrombus and a mixed inflammatory cell infiltrate. Many other neurological signs, suggesting the widespread involvement of the central nervous system, had already been observed through a rigorous clinical evaluation. These included convulsions, blindness, signs of vestibular and cerebellar involvement (tilted head, nystagmus, motor incoordination, and tremors), paraparesis, tetraparesis, tetraplegia and myoclonia [11].

Therefore, the aim of this study was to evaluate histopathological alterations and verify the presence of $L$. infantum in specific areas of the encephalon of naturally infected dogs, in order to verify if this parasite results in tropism in a certain region of the central nervous system.

\section{Methods}

Animals

This study was performed at the Veterinary Hospital of the Universidade Estadual do Maranhão, Brazil, in accordance with the Ethics Committee on Animal Experimentation (protocol number 05/2006). The dogs owners signed and informed consent form. Fifteen male and female mongrel dogs from São Luís Island, an L. infantum endemic area took a part of this experiment. All animals were positive for L. infantum for direct parasitological examination (bone marrow aspiration) and serological test (ELISA). After clinical evaluation, the animals received $2 \%$ xylazine as premedication $(0.1 \mathrm{~mL} / \mathrm{kg}$ intravenously) and $2.5 \%$ thiopental as an anesthetic. After a latency period of $5 \mathrm{~min}$, euthanasia was performed by injecting $10 \%$ potassium chloride intravenously. Necropsy was then carried out for brain removal.

\section{Histopathological and immunohistochemistry analysis}

The entire encephala of the dogs were removed and fixed in $10 \%$ buffered formalin for $24 \mathrm{~h}$. After that, fragments of the cerebellum, cerebellar peduncle, rostral colliculus, caudal colliculus, thalamus, hippocampus, and telencephalon were obtained, submitted to histological processing, and stained by the standard Hematoxilyn-eosin method. For immunohistochemical analysis, sections of $5 \mu \mathrm{m}$ were fixed on silanized slides and the streptavidin-peroxidase method was performed [12].

\section{DNA extraction from paraffin-embedded samples}

Sections $(10 \mu \mathrm{m})$ of each tissue sample were collected in microtubes to remove paraffin with xylol. Next, the tissues were rehydrated using decreasing concentrations of ethanol and the supernatant was removed. A total of $200 \mu \mathrm{L}$ of lysis buffer and $20 \mu \mathrm{L}$ of proteinase $\mathrm{K}(20 \mathrm{mg} / \mathrm{ml})$ were added as described by Pikor et al. [13]. The extraction was carried out using the phenol-chloroform-isoamylic alcohol method. The extracted DNA samples were resuspended in $15 \mu \mathrm{L}$ of ultrapure water and stored at $-20{ }^{\circ} \mathrm{C}$ until use as previously described by de Lima [14].

\section{Polymerase chain reaction (PCR)}

The extracted DNA was amplified in a thermocycler using the primers RV1 (sense; 5'-CTTTTCTGGT CCCGCGGGTAG-3') and RV2 (antisense; 5'CCACC TGGCCTATTTTACACCA-3'). This targets a conserved region of $L$. (L.) infantum kDNA and produces a fragment of approximately 145 base pairs. Positive and negative controls were used in each reaction. The PCR products were analyzed on $1 \%$ agarose gel with a molecular weight marker of $100 \mathrm{bp}(1 \mathrm{~Kb}$ DNA ladder Promega $^{\oplus}$ [15].

\section{Isolation and characterization of the parasites}

Parasites were isolated from bone marrow and cultivated in Novy Macneal Nicolle medium (NNN) and posteriorly sent to Laboratory of Immunomodulation and Protozoology/ IOC - FIOCRUZ, incubated at 26 to $28{ }^{\circ} \mathrm{C}$ and examined weekly. Positive cultures were sent to the National Reference Laboratory for Leishmania (CLIOC) of the Oswaldo Cruz Institute to characterize the Leishmania species using the enzyme electrophoresis technique [16].

\section{Results}

The isolated parasites were characterized by the technique of isoenzyme electrophoresis as identified $L$. infantum. The animals used in the present study displayed at least one clinical sign compatible with visceral leishmaniasis, only one animal showed signs of neurological disease. The most frequently observed clinical signs were cutaneous lesions (86.6\%) and cachexia (66.6\%). In post-mortem evaluation, hepatosplenomegaly and lymphadenopathy, present in $86.6 \%$ of the dogs, were the most frequent alterations.

Histopathological analysis revealed that $93.3 \%$ of the animals had lesions in at least two of the analyzed brain regions. These lesions were distributed in the telencephalon, hippocampus, rostral colliculus and cerebellum. Lymphoplasmocitary inflammatory reaction was the most frequent lesion (37.1\%) associated with rare amastigotes, Gitter cells also were frequent among inflammatory cells (Fig. 1. hemorrhages (10.4\%), gliosis (4.7\%) and chromatolysis $(3.8 \%)$ were also observed. 


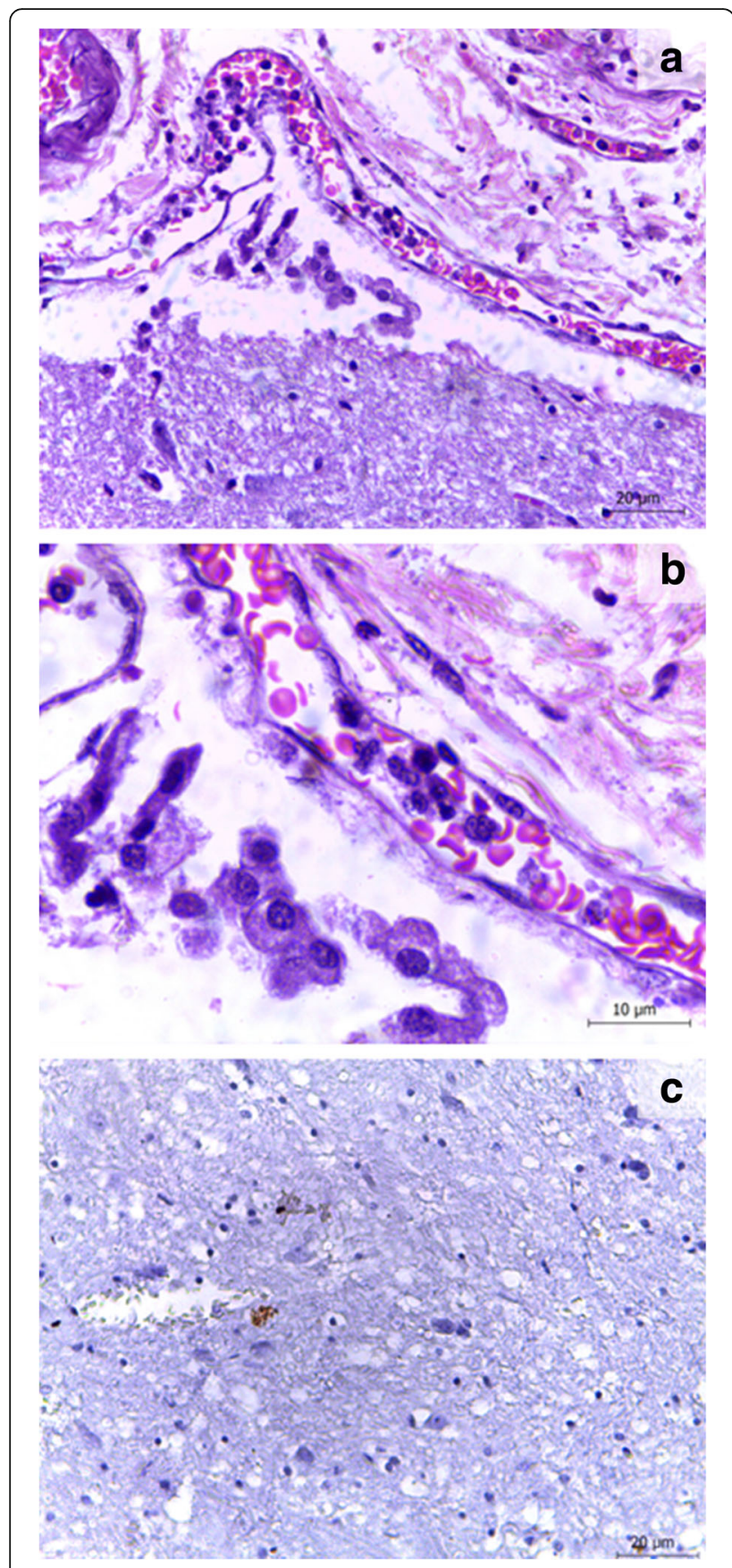

Fig. 1 a Microscopic lesions in cerebellum. Encephalon of dog with visceral leishmaniasis presenting inflammatory infiltrate with foamy macrophages or Gitter cell in the cerebellum region (bar $20 \mu \mathrm{m}$ ): b higher magnification (bar $10 \mu \mathrm{m}$ ). Hematoxylin and eosin stain. c Encephalon of dog with visceral leishmaniasis. Rostral colliculus region marked amastigote forms (arrow) Immunohistochemistry (bar $20 \mu \mathrm{m}$ )

Immunohistochemical analysis detected amastigote forms in seven dogs. The amastigotes were associated with an inflammatory reaction in all the animals (Fig. 1c). Parasites were found in four different regions: the thalamus $(n=3)$, telencephalon $(n=3)$, hippocampus $(n=2)$ and caudal colliculus $(n=2)$.

Polymerase chain reaction detected parasite DNA in 11 samples from seven dogs. The positive encephalic regions were the telencephalon $(n=3)$; thalamus $(n=2)$, hippocampus $(n=2)$; cerebellum $(n=2)$; caudal colliculus $(n=1)$ and rostral colliculus $(n=1)$. All positive samples in PCR also presented some histopathological changes, such as inflammatory reaction composed by mononuclear cells.

\section{Discussion}

During Leishmania infection the BBB may be damaged, allowing that amastigotes reach brain parenchyma, where an intensive inflammatory reaction can be observed. The mechanisms by which this process occurs remain unknown, however, some intracellular bacteria, such as Listeria monocytogenes seem to cross the BBB inside leukocytes, a mechanism known as "Trojan horse" [17, 18]. Abreu-Silva et al. [8] suggest that Leishmania uses this strategy to penetrate the brain parenchyma.

Inflammatory reaction during canine visceral leishmaniasis was also observed by Ikeda et al. [11], who analyzed brains of dogs with and without neurological signs and described degenerative alterations with neuronophagia, gliosis, leptominingitis, vascular congestion and the presence of a perivascular lymphoplasmacytic infiltrate and areas of micro-hemorrhages.

Melo et al. [19] evaluated the presence of leukocytes in the brains of dogs with VL, and observed significant inflammatory lesions consisting of lymphocytes, macrophages, plasma cells and some neutrophils. An immunohistochemical study showed that CD3+ T lymphocytes are important components of the inflammatory infiltrate present in the choroid plexus, meninges and perivascular brain parenchyma. Moreover, the cytokine profile identified high expressions of IL- $1 \beta$ and TNF- $\alpha$, which are considered key factors for the beginning, maintenance and persistence of inflammation [20].

Leishmania amastigotes in the CNS of dogs with visceral leishmaniasis were previously described by [21, 22]. Other studies, despite the observation of histopathological alterations in the CNS of dogs during visceral leishmaniasis, failed to verify the presence of Leishmania amastigotes [11, 19, 20, 23-25]. Some authors explain these contradictory findings by the chronicity of the disease, arguing that the presence of Leishmania in the CNS has generally been demonstrated in dogs with an earlier infection, either because of delayed diagnosis or by the choice of treatment for the infected dogs, as in two studies in the Mediterranean region [11, 24]. In fact, here we observed that animals that presented amastigote forms in the CNS had clinical signs such as 
cachexia and the presence of skin lesions, observed in the chronic phase.

\section{Conclusion}

Dogs naturally infected $L$. infantum can present alterations in the CNS due mononuclear inflammatory infiltrate. Furthermore, Leishmania parasites have unknown mechanisms that allow their entrance into the CNS, and show no preference for a specific region of the system.

\section{Abbreviations}

BBB: Blood-brain barrier; CNS: Central nervous system; CSF: Cerebrospinal fluid; CVL: Canine visceral leishmaniasis; PCR: Polymerase chain reaction

\section{Acknowledgements}

The authors thank to FAPEMA and CNPq for financial support.

\section{Funding}

This work was funded by Fundação de Amparo à Pesquisa e Desenvolvimento Científico do Maranhão (APP-00844/09 and Pronex-241,709/2014) and Conselho Nacional de Desenvolvimento Científico e Tecnológico (CNPq).

\section{Availability of data and materials}

All data generated or analyzed during this study are included in the manuscript.

\section{Authors' contributions}

All the authors had full access to the complete study data and take responsibility for the integrity of data and accuracy of data analysis. Study concept and design: ALAS. Data acquisition: WLM, JCSá, APCS, ALR and RMO. Analysis and interpretation of data: ALAS, APCS and WLM. Drafting of the manuscript: ALAS and WLM. Critical revision of the manuscript for significant intellectual content: JCS, FHEA and KSC. Obtained funding: ALAS. Administrative, technical, and material support: FHEA. Necropsy and isolation and identification of Leishmania -CMC and KSC. Study supervision: ALAS. All authors read and approved the final manuscript.

\section{Ethics approval}

This study was approved by Ethics Committee on Animal Experimentation of Universidade Estadual do Maranhão (protocol number 05/2006).

\section{Consent for publication}

All authors give their consent for the article publication.

\section{Competing interests}

The authors declare that they have no competing interests.

\section{Publisher's Note}

Springer Nature remains neutral with regard to jurisdictional claims in published maps and institutional affiliations.

\section{Author details}

${ }^{1}$ Center for Biological and Health Sciences, Universidade Federal do Maranhão, São Luis, MA CEP 65080-805, Brazil. ${ }^{2}$ Medicine Coordination, Universidade CEUMA, São Luís, MA CEP 65055-000, Brazil. 'Veterinary School, Universidade Federal do Tocantins, Araguaína, TO CEP 77804-970, Brazil. ${ }^{4}$ Department of Pathology, Universidade Estadual do Maranhão, São Luís, MA CEP 65055-000, Brazil. 'Laboratory of Immunomodulation and Protozoology, Instituto Oswaldo Cruz, Rio de Janeiro, Brazil.

\section{Received: 6 September 2016 Accepted: 9 August 2017}

\section{Published online: 18 August 2017}

\section{References}

1. Alvar J, Cañavate C, Molina R, Moreno J, Nieto J. Canine leishmaniasis. Adv Parasitol. 2004:57:1-88.

2. Baneth G, Koutinas AF, Solano-Gallego L, Bourdeau P, Ferrer L. Canine leishmaniasis - new concepts and insights on an expanding zoonosis: part one. Trends Parasitol. 2008;24:324-30.
3. Giunchetti RC, Martins-Filho OA, Carneiro CM, Mayrink W, Marques MJ, Tafuri WL, et al. Histopathology, parasite density and cell phenotypes of the popliteal lymphnode in canine visceral leishmaniasis. Vet Immunol Immunopathol. 2008;121:23-33.

4. Giunchetti RC, Mayrink W, Carneiro CM, Corrêa-Oliveira R, Martins-Filho OA, Marques MJ, et al. Histopathological and immunohistochemical investigations of the hepatic compartment associated with parasitism and serum biochemical changes in canine visceral leishmaniasis. Res Vet Sci. 2008;84:269-77.

5. Persidsky Y, Ramirez SH, Haorah J, Kanmogne GD. Blood-brain barrier: structural components and function under physiologic and pathologic conditions. J Neurolmmune Pharmacol. 2006;1(3):223-36.

6. Prasad LS, Sen S. Migration of Leishmania donovani amastigotes in the cerebrospinal fluid. Am. J. Trop. Med. Hyg. 1996;55:652-4.

7. Garcia-Alonso M, Nieto CG, Blanco A, Requena JM, Alonso C, Navarrete I. Presence of antibodies in the aqueous humour and cerebrospinal fluid during Leishmania infections in dogs. Pathological features at the central nervous system. Parasite Immunol. 1996;18:539-46.

8. Abreu-Silva AL, Calabrese KS, Tedesco RC, Mortara RA, Costa SCG. Central nervous system involvement in experimental infection with Leishmania (Leishmania) amazonensis. Am J Trop Med Hyg. 2003;68:661-5.

9. Oliveira E, Oshiro ET, Pinto R, Castro BC, Daniel KB, Oliveira JM, et al. Presence of amastigotes in the central nervous system of hamsters infected with Leishmania sp. Rev Bras Parasitol Vet. 2011;20(2):97-102.

10. Font A, Mascort J, Altimira J, Closa JM, Vilafranca M. Acute paraplegia associated with vasculitis in a dog with leishmaniasis. J Small Anim Pract. 2004:45:199-201.

11. Ikeda FA, Laurenti MD, Corbett CE, Feitosa MM, Machado GF, Perry SHV. Histological and immunohistochemical study of the central nervous system of dogs naturally infected by Leishmania (Leishmania) infantum. Braz J Vet Res Anim Sci. 2007:44:5-11.

12. Tafuri WL, Santos RL, Arantes RME, Gonçalves R, Melo MN, Michalik MSM, Tafuri WL. An alternative immunohistochemical method for detecting Leishmania amastigotes in paraffin-embedded canine tissues. J Immunol Methods. 2004;292:17-23.

13. Pikor LA, Enfield KSS, Cameron H, Lam WL. DNA extraction from paraffin embedded material for genetic and epigenetic. Jo VE. 2011;108:1-3.

14. de Lima AC, Zampieri RA, Tomokane TY, Laurenti MD, Silveira FT, Corbett CE, Floeter-Winter LM, Gomes CM. Leishmania sp. identification by PCR associated with sequencing of target SSU rDNA in paraffin-embedded skin samples stored for more than 30 years. Parasitol Res. 2011;108:1525-31.

15. Lachaud L, Marchergui-Hammami S, Chabbert E, Dereure J, Dedet JP, Bastien P. Comparison of six PCR methods using peripheral blood for detection of canine visceral Leishmaniasis. J Clin Microbiol. 2002;40:210-5.

16. Cupolillo E, Grimaldi G Jr, Momen H. A general classification of new world Leishmania using numerical zymotaxonomy. Am J Trop Med Hyg. 1994;50:296-311.

17. Drevets DA. Dissemination of Listeria monocytogenes by infected phagocytes Infect. Immun. 1999;67:3512-7.

18. Masocha W, Kristensson K. Passage of parasites across the blood-brain barrier. Virulence. 2012;3:202-12.

19. Melo GD, Machado GF. Choroid plexus involvement in dogs with spontaneous visceral leishmaniasis: a histopathological investigation. Braz J Vet Pathol. 2009;2:69-74.

20. Melo GD, Seraguci TF, Schweigert A, Silva JES, Grano FG, Peiró JR, et al. Pro-inflammatory cytokines predominate in the brains of dogs with visceral leishmaniasis: a natural model of neuroinflammation during systemic parasitic infection. Vet Parasitol. 2013;192:57-66.

21. Viñuelas J, García-Alonso M, Ferrando L, Navarrete I, Molano I, Mirón C, et al. Meningeal leishmaniasis induced by Leishmania infantum in naturally infected dogs. Vet Parasitol. 2001;101:23-7.

22. Marquez M, Pedregosa JR, López J, Marco-Salazar P, Fondevila D, Pumarola M. Leishmania amastigotes in the central nervous system of a naturally infected dog. J Vet Diagn Investig. 2012;25(1):142-6.

23. Lima VMF, Gonçalves ME, Ikeda FA, Luvizotto MCR, Feitosa MM. Anti-Leishmania antibodies in cerebrospinal fuid from dogs with visceral leishmaniasis. Bras J Med Bio Res. 2003:36:485-9.

24. Melo GD, Marcondes M, Vasconcelos RO, Machado GF. Leukocyte entry into the CNS of Leishmania chagasi naturally infected dogs. Vet Parasitol. 2009:162:248-56.

25. Melo GD, Machado GF. Glial reactivity in dogs with visceral leishmaniasis: correlation with T lymphocyte infiltration and with cerebrospinal fluid anti-Leishmania antibody titres. Cell Tissue Res. 2011;346:293-304. 